\title{
Serum matrix metalloproteinase-9 level in systemic lupus erythematosus with peripheral neuropathy
} Mohamed Hassan Imam ${ }^{a}$, HamdyKhamis Koriem ${ }^{a}$, Marwa Mohamed Hassan ${ }^{a}$, Abeer Shawky El-Hadidi ${ }^{\mathrm{b}}$, Niveen Abdallah Ibrahim $^{\mathrm{a}}$

\author{
aDepartment of Physical Medicine, \\ Rheumatology and Rehabilitation clinic, Faculty \\ of Medicine, Alexandria University, Egypt, \\ bepartment of Clinical and Chemical \\ Pathology, Faculty of Medicine, Alexandria \\ University, Egypt \\ Correspondence to Dr. Niveen Abdallah \\ Ibrahim Ahmed, MBBCh, Master of Physical \\ Medicine, Rheumatology and Rehabilitation, \\ Department of Physical Medicine, Faculty of \\ Medicine, Alexandria University, Egypt. \\ Tel: +201223797072; \\ e-mail: niveenabbdallah@gmail.com
}

Received 28 May 2019

Accepted 17 July 2019

Egyptian Rheumatology \& Rehabilitation 2019, 46:211-220

\begin{abstract}
Objective
To evaluate whether serum matrix metalloproteinase-9 (MMP-9) is associated with peripheral neuropathy (PN) in patients with systemic lupus erythematosus (SLE) and to determine the relationship between MMP-9 serum level and SLE disease activity, lupus manifestations, and laboratory markers.

Patients and methods

A total of 30 patients with SLE with PN, 30 patients with SLE without PN, and 20 healthy controls were included in this study. SLE clinical manifestations, Systemic Lupus Activity Measure (SLAM) index, and laboratory markers were evaluated. All the data were compared and correlated with serum MMP-9 level.

Results

MMP-9 showed a significant increase in frequency in SLE with PN group compared with SLE without PN group $\left(P_{1}=0.037\right)$, SLE with PN group compared with control group $\left(P_{2}<0.001\right)$, and SLE without PN group compared with control group $\left(P_{3}<0.001\right)$. In comparison between SLE with normal MMP-9 group versus SLE with high MMP-9 group, it showed no statistically significant difference between the two groups regarding demographic data, SLAM index, Erythrocytes sedimentation rate (ESR), C-reactive protein (CRP), Antinuclear antibodies (ANA), Antiphospholipid antibodies (APL), C3, C4, anti-double-stranded DNA, and lupus clinical features, except malar rash and lupus nephritis, which showed significant increase in SLE with high MMP-9 group compared with SLE with normal MMP-9 group ( $P=0.042$ for each). A significant positive correlation was detected between MMP-9 serum level and SLAM index $(P=0.037)$, whereas antidouble-stranded DNA did not show significant correlation. There was a significant relation between increasing the risk of PN and MMP-9 (odds ratio=4.031).

Conclusion

Significant elevation of serum MMP-9 may increase the risk of PN in patients with SLE, and it may correlate with disease activity, lupus nephritis, and skin involvement.

\footnotetext{
Keywords:

Egypt Rheumatol Rehabil 46:211-220

(c) 2019 Egyptian Society for Rheumatology and Rehabilitation

1110-161X
}

matrix metalloproteinase-9, peripheral neuropathy, systemic lupus erythematosus
\end{abstract}

\section{Introduction}

Matrix metalloproteinases (MMPs) are a group of zinccontaining endoproteinases that can corrupt an assortment of extracellular matrix components [1]. MMP-9 is a kind of gelatinase that is secreted by certain cell types of the vessel wall, including $T$ lymphocytes, macrophages, endothelial cells, as well as smooth muscle cells [2]. The main role of T cell-derived MMP-9 is to facilitate T-cell migration through connective tissue [3]. Increased activity of MMPs has been involved in various illness forms, including cardiovascular diseases and autoimmune diseases such as multiple sclerosis, Guillain-Barré syndrome, systemic lupus erythematosus (SLE), rheumatoid arthritis, and malignancies [4,5], for instance, MMPs have a role in other rheumatological diseases, as elevated serum and synovial MMP-3 levels reflect disease activity in patients with rheumatoid arthritis [6].
Multiple studies analyzed MMP-9 activity in the sera of patients with active and inactive SLE to evaluate its ease as well as its diagnostic value. MMP-9 activity was significantly higher in the sera of patients with SLE compared with those of healthy controls [7-9]. The high levels of MMP-9 and its correlation to disease activity indices are controversial [10-12].

In another study, circulating MMP-9 serum levels were compared with antibodies to single-stranded DNS and double-stranded DNA (ssDNA and dsDNA), so as to decide their relationship to inflammatory arthritic diseases. Patients having high

\footnotetext{
This is an open access journal, and articles are distributed under the terms of the Creative Commons Attribution-NonCommercial-ShareAlike 4.0 License, which allows others to remix, tweak, and build upon the work non-commercially, as long as appropriate credit is given and the new creations are licensed under the identical terms.
} 
anti-ssDNA and anti-dsDNA antibodies harbored significantly lower amounts of MMP-9 when compared with patients who had low levels of both anti-ssDNA and anti-dsDNA antibodies. Hence, neutrophil MMP-9, an inflammatory marker, inversely correlates with anti-dsDNA antibodies. The presence of antibodies, or another compound in patients with SLE might hinder the synthesis of MMP-9 or its activity. Another conceivable clarification is that the calcium ions are inhibited or destroyed, and in this way activated MMP-9 is being degraded [9].

With respect to neuropsychiatric SLE, it has been proposed that autoantibody-mediated neuronal cell damage in SLE brains may be either a direct consequence of the vasculopathy or, alternatively, be owing to altered blood-brain barrier permeability for the autoantibodies. This raises the issue of whether altered endoproteinase expression plays a role in the vascular harm in both central nervous system (CNS) and peripheral nervous system (PNS) [13].

It has been demonstrated that infiltration of the nerve tissue by macrophages and $\mathrm{T}$ cells across a functionally disturbed blood-nerve barrier (BNB) is one of the evident pathophysiological signs of inflammationmediated peripheral neuropathies. Among the effector molecules that might play a role in BNB function disturbance are MMPs. MMP-9 is capable of degrading the subendothelial basement membrane, and may in this manner be involved in the disturbance of the BNB. Moreover, altered expression of MMPs in the vessel walls might be an additional mechanism for blood vessel damage, resulting in ischemic nerve damage. Support for this theory comes from recent findings that plasma levels of MMP-9 are higher in patients with SLE $[10,14]$. In this way, the upregulation of MMP-9 within the vessel walls may be responsible for the vascular damage seen in SLE and the resulting chronic combined axonal and demyelinating type of neuropathy frequently found in SLE [13]; thus, further studies are needed to evaluate and test the theory that serum MMP-9 levels are associated with peripheral neuropathy $(\mathrm{PN})$ in patients with SLE.

\section{Patients and methods}

A total of 60 patients were selected from those attending the Outpatient Clinic of Physical Medicine, Rheumatology and Rehabilitation Department and Rheumatology Outpatient Clinic, and Internal Medicine Department, the Main
Alexandria University Hospital. They were divided into three groups:

This study was carried out on three groups of patients:

Group I (SLE with PN): it included 30 adult patients with SLE, fulfilling the Systemic Lupus International Collaborating Clinics (SLICC) criteria [15], along with peripheral neuropathies. Definitions of peripheral neuropathies were provided by the American college of rheumatology (ACR) nomenclature and case definitions for neuropsychiatric syndromes in patients with SLE [16].

The following were the clinically exclusion criteria:

(1) Patients aged less than 18 years old.

(2) Patients with connective tissue diseases.

(3) Patients with other overt causes of PN such as follows:

(a) Endocrinal: for example, diabetes mellitus and hypothyroidism.

(b) Metabolic: such as renal and liver failure.

(c) Drugs and toxins, for example, alcohol abuse, vincristine, metronidazole, phenytoin, isoniazid, and fluoroquinolone.

(d) Compression neuropathy.

(e) Traumatic neuropathy.

(f) Infection: Herpes zoster and leprosy.

(g) Malignancy.

Group II (SLE without PN): it included 30 adult patients with SLE fulfilling the SLICC criteria [15] without clinical manifestations of PN and confirmed by normal electrophysiological study.

Groups I and II were subjected to the following:

(1) Recording of historical data for patients, including personal, present, past, family, drug, and obstetrical history.

(2) Registration of anthropometric measures.

(3) Thorough clinical examination including neurological and musculoskeletal examination.

(4) Systemic Lupus Activity Measure (SLAM) index [17].

(5) Electrophysiological assessment, including motor conduction study of median, ulnar, tibial, and common peroneal nerves at both sides. Sensory conduction study of median, ulnar, and sural nerves at both sides. Bilateral blink reflex and sympathetic skin response test was examined in both hands.

(6) Laboratory assessment, including complete blood count $(\mathrm{CBC})$, erythrocytes sedimentation rate 
(ESR), C-reactive protein (CRP), serum creatinine, urea, complete urine analysis, Antinuclear antibodies (ANA), anti-dsDNA, C3, C4, and serum (MMP-9) using quantitative technique. Serum MMP-9 was tested for all groups.

Group III: 20 healthy individual as a control group, matched for age and sex with the case group.

\section{Statistical analysis}

Data were fed to the computer and analyzed using IBM SPSS software package version 20.0. (IBM Corp., Armonk, New York, USA). Qualitative data were described using number and percent. The Kolmogorov-Smirnov test was used to verify the normality of distribution. Description of the quantitative data was done using the mean $\pm S D$ in normally distributed data, whereas using the medium (the minimum and the maximum) in not normally distributed one. Significance of the obtained results was judged at the 5\% level. Comparison between different groups regarding categorical variables was tested using $\chi^{2}$-test; when more than $20 \%$ of the cells have expected count less than 5 , corrections for $\chi^{2}$ was conducted using Fisher's exact test. Student $t$-test was used for normally distributed quantitative variables, to compare between two studied groups, whereas Mann-Whitney test was used for abnormally distributed quantitative variables. Odds ratio was used to calculate the ratio of the odds and 95\% confidence interval of an event occurring in one risk group (SLE with $\mathrm{PN}$ ) to the odds of it occurring in the nonrisk group (SLE without PN). Regression analysis was done to detect the most independent/affecting factor for PN. Distributions of the different electrophysiological parameters were calculated at 5 th and 95 th percentiles.

Receiver operating characteristic curve (ROC) is generated by plotting sensitivity (true positive) on $y$ axis versus 1 -specificity (false positive) on $x$-axis at different cutoff values. The area under the ROC curve denotes the diagnostic performance of the test. Area more than $50 \%$ gives acceptable performance and area of $\sim 100 \%$ is the best performance for the test. The ROC curve allows also a comparison of performance between two tests.

\section{Results}

This study recruited 60 (30 with PN and 30 without PN) patients with SLE, with an age range of 18-58 years, and 10 controls, with an age range of 20-44 years. The duration of the disease ranged from 3 to 240 months.

Comparison among the three studied groups regarding high serum matrix metalloproteinase-9 level

Regarding the frequency of high serum MMP-9 level in group I, 26 (86.7\%) patients had high, whereas four (13.3\%) patients were normal; in group II, 19 (63.3\%) patients had high, whereas $11(36.7 \%)$ patients were normal; and in group III, one (5\%) patient had high, whereas $19(95 \%)$ patients were normal. The number of patients with high serum MMP-9 level was statistically higher in group I compared with group II $\left(P_{1}=0.037\right)$, and also there was a statistically significant increase in the number of patients with high serum MMP-9 levels in group I compared with group III $\left(P_{2}<0.001\right)$, and similarly in group II compared with group III $\left(P_{3}<0.001\right.$; Table 1 and Fig. 1$)$.

The 60 patients with SLE were subdivided into two groups:

(1) SLE with normal serum MMP-9 group (15 patients).

(2) SLE with high serum MMP-9 group (45 patients).

Comparison between systemic lupus erythematosus with normal serum matrix metalloproteinase-9 group and systemic lupus erythematosus with high serum matrix metalloproteinase-9 group regarding demographic data

There was no statistically significant difference between the two studied groups regarding sex, age, height, and BMI (Table 2).

Table 1 Comparison among the three studied groups regarding high serum matrix metalloproteinase-9 level

\begin{tabular}{lcccc}
\hline & $\begin{array}{c}\text { SLE with PN }(n=30)[N \\
(\%)]\end{array}$ & $\begin{array}{c}\text { SLE without PN }(n=30)[N \\
(\%)]\end{array}$ & $\begin{array}{c}\text { Control }(n=20)[N \\
(\%)\end{array}$ & $\chi^{2}$ \\
\hline Matrix metalloproteinase-9 & & & $P$ \\
$\quad$ Normal $(\leq 15.7)$ & $4(13.3)$ & $11(36.7)$ & $19(95.0)$ & $33.419^{*}<0.001^{*}$ \\
$\quad$ High $(>15.7)$ & $26(86.7)$ & $19(63.3)$ & $1(5.0)$ & \\
$\begin{array}{l}\text { Significance between the } \\
\text { groups }\end{array}$ & & $P_{1}=0.037^{\star}, P_{2}<0.001^{*}, P_{3}<0.001^{*}$ & & \\
\hline
\end{tabular}

$P, P$ value for comparing the three groups; $\mathrm{PN}$, peripheral neuropathy; $\mathrm{SLE}$, systemic lupus erythematosus. $P_{1}: P$ value for comparing from SLE with PN and SLE without PN. $P_{2}: P$ value for comparing from SLE with PN and control. $P_{3}: P$ value for comparing from SLE without $P N$ and control. " $P \leq 0.05$, statistically significant. 
Figure 1

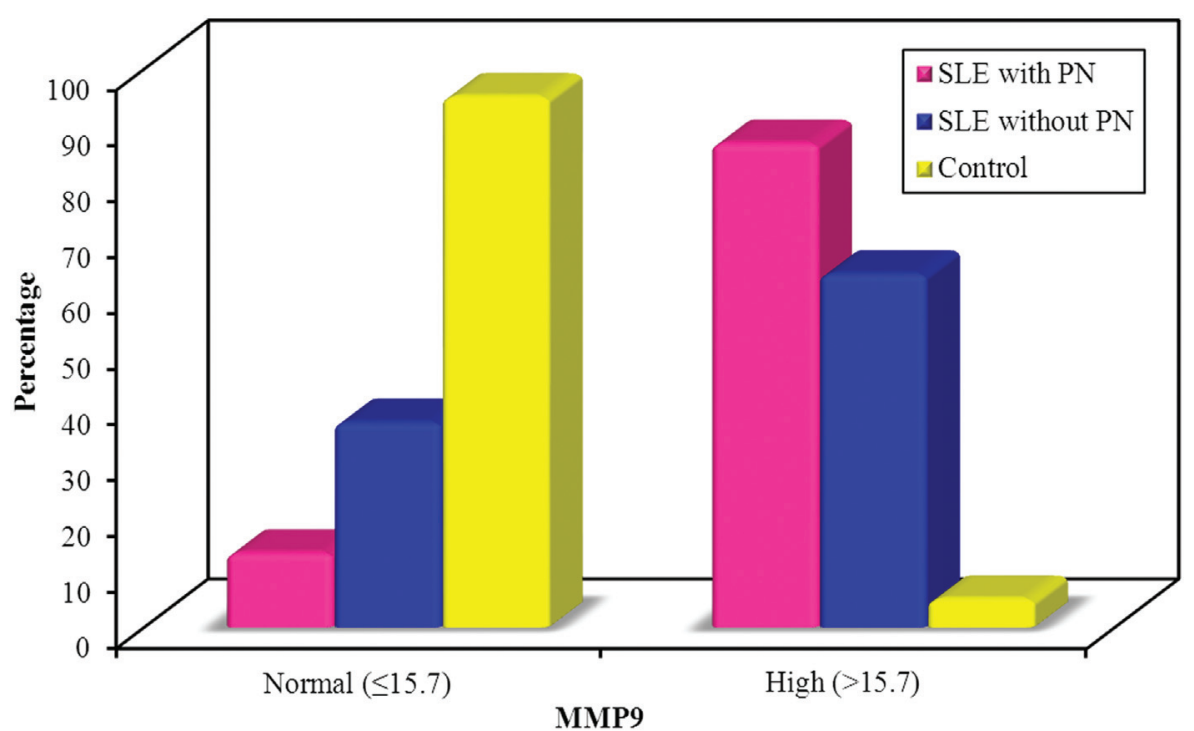

Comparison among the three studied groups regarding high serum matrix metalloproteinase-9 level.

Table 2 Comparison between systemic lupus erythematosus with normal serum matrix metalloproteinase-9 group and systemic lupus erythematosus with high serum matrix metalloproteinase-9 group regarding demographic data $(n=60)$

\begin{tabular}{|c|c|c|c|c|}
\hline & \multicolumn{2}{|c|}{ Matrix metalloproteinase-9 $[n(\%)]$} & \multirow[t]{2}{*}{ Test of significance } & \multirow[t]{2}{*}{$P$} \\
\hline & Normal $(\leq 15.7)(n=15)$ & High $(>15.7)(n=45)$ & & \\
\hline \multicolumn{5}{|l|}{ Sex } \\
\hline Female & $12(80.0)$ & $41(91.1)$ & $\chi^{2}=1.348$ & ${ }^{\mathrm{FE}} P=0.351$ \\
\hline Male & $3(20.0)$ & $4(8.9)$ & & \\
\hline \multicolumn{5}{|l|}{ Age (years) } \\
\hline Range & $18.0-58.0$ & $18.0-55.0$ & $t=0.278$ & 0.784 \\
\hline Mean \pm SD & $36.13 \pm 12.65$ & $37.11 \pm 8.67$ & & \\
\hline \multicolumn{5}{|l|}{ Weight (kg) } \\
\hline Range & $56.0-89.50$ & $40.0-91.0$ & $t=1.005$ & 0.319 \\
\hline Mean \pm SD & $74.83 \pm 10.98$ & $71.46 \pm 11.36$ & & \\
\hline \multicolumn{5}{|l|}{ Height $(\mathrm{cm})$} \\
\hline Range & $1.55-1.76$ & $1.50-1.76$ & $t=0.527$ & 0.600 \\
\hline Mean \pm SD & $1.64 \pm 0.07$ & $1.63 \pm 0.06$ & & \\
\hline \multicolumn{5}{|l|}{ BMI $\left(\mathrm{kg} / \mathrm{m}^{2}\right)$} \\
\hline Range & $21.13-35.41$ & $16.60-33.90$ & $t=0.842$ & 0.403 \\
\hline Mean \pm SD & $27.91 \pm 4.38$ & $26.85 \pm 4.16$ & & \\
\hline
\end{tabular}

FE, Fisher exact; $P, P$ value for comparing between the two categories.

Comparison between systemic lupus erythematosus with normal serum matrix metalloproteinase-9 group and systemic lupus erythematosus with high serum matrix metalloproteinase-9 group regarding systemic lupus erythematosus clinical features

There were no statistically significant differences between the two studied groups (SLE with normal serum MMP-9 group versus SLE with high serum MMP-9 group), regarding SLE clinical features, except of malar rash and lupus nephritis.

In SLE with normal serum MMP-9 group, two (13.3\%) patients had malar rash, in comparison with
19 (42.2\%) patients in SLE with high MMP-9 group. Malar rash showed a significant increase in SLE with high serum MMP-9 group compared with SLE with normal serum MMP-9 group (Table 3 and Fig. 2).

In SLE with normal serum MMP-9 group, two (13.3\%) patients had nephritis, in comparison with 19 (42.2\%) patients in SLE with high serum MMP9 group. Nephritis showed a significant increase in SLE with high serum MMP-9 group compared with SLE with normal serum MMP-9 group (Table 3 and Fig. 2). 
Table 3 Comparison between systemic lupus erythematosus with normal serum matrix metalloproteinase-9 group and systemic lupus erythematosus with high serum matrix metalloproteinase-9 group regarding systemic lupus erythematosus clinical features $(n=60)$

\begin{tabular}{|c|c|c|c|c|}
\hline \multirow[t]{2}{*}{ Clinical features } & \multicolumn{2}{|c|}{ Matrix metalloproteinase-9 $[n(\%)]$} & \multirow[t]{2}{*}{$\chi^{2}$} & \multirow[t]{2}{*}{$P$} \\
\hline & Normal $(\leq 15.7)(n=15)$ & High $(>15.7)(n=45)$ & & \\
\hline Fever & $3(20.0)$ & $14(31.1)$ & 0.684 & ${ }^{\mathrm{FE}} P=0.520$ \\
\hline Fatigue & $13(86.7)$ & $45(100.0)$ & 6.207 & ${ }^{\mathrm{FE}} P=0.059$ \\
\hline Malar rash & $2(13.3)$ & $19(42.2)$ & $4.127^{\star}$ & $0.042^{*}$ \\
\hline Chronic cutaneous & $3(20.0)$ & $7(15.6)$ & 0.160 & ${ }^{\mathrm{FE}} P=0.700$ \\
\hline Photosensitivity & $11(73.3)$ & $38(84.4)$ & 0.928 & ${ }^{\mathrm{FE}} P=0.442$ \\
\hline Alopecia & $11(73.3)$ & $40(88.9)$ & 2.135 & ${ }^{\mathrm{FE}} P=0.208$ \\
\hline Oral ulcers & $13(86.7)$ & $30(66.7)$ & 2.216 & ${ }^{\mathrm{FE}} P=0.192$ \\
\hline Arthralgia/arthritis & $13(86.7)$ & $42(93.3)$ & 0.655 & ${ }^{\mathrm{FE}} P=0.591$ \\
\hline Raynaud's phenomenon & $2(13.3)$ & $3(6.7)$ & 0.655 & ${ }^{\mathrm{FE}} P=0.591$ \\
\hline Vasculitis & $1(6.7)$ & $7(15.6)$ & 0.769 & ${ }^{\mathrm{FE}} P=0.666$ \\
\hline Serositis & $2(13.3)$ & $5(11.1)$ & 0.054 & ${ }^{\mathrm{FE}} P=1.000$ \\
\hline Nephritis & $2(13.3)$ & $19(42.2)$ & $4.127^{*}$ & $0.042^{*}$ \\
\hline Pneumonia & $2(13.3)$ & $5(11.1)$ & 0.054 & ${ }^{\mathrm{FE}} P=1.000$ \\
\hline \multicolumn{5}{|l|}{ CNS } \\
\hline Headache & $3(20.0)$ & $18(40.0)$ & 1.978 & 0.160 \\
\hline Seizures & $1(6.7)$ & $1(2.2)$ & 0.690 & ${ }^{\mathrm{FE}} P=0.441$ \\
\hline Psychosis & 0 & $4(8.9)$ & 1.429 & ${ }^{\mathrm{FE}} P=0.564$ \\
\hline Cognitive deficit & 0 & $2(4.4)$ & 0.690 & ${ }^{\mathrm{FE}} P=1.000$ \\
\hline CVS & $1(6.7)$ & $2(4.4)$ & 0.117 & ${ }^{\mathrm{FE}} P=1.000$ \\
\hline Transverse myelitis & $1(6.7)$ & $1(2.2)$ & 0.690 & ${ }^{\mathrm{FE}} P=0.441$ \\
\hline \multicolumn{5}{|l|}{ Hematological disease } \\
\hline Anemia & $9(60.0)$ & $25(55.6)$ & 0.090 & 0.764 \\
\hline Leukopenia & $4(26.7)$ & $8(17.8)$ & 0.556 & ${ }^{\mathrm{FE}} P=0.472$ \\
\hline Thrombocytopenia & $2(13.3)$ & $5(11.1)$ & 0.054 & ${ }^{\mathrm{FE}} P=1.000$ \\
\hline Pancytopenia & 0 & $3(6.7)$ & 1.053 & ${ }^{\mathrm{FE}} P=0.566$ \\
\hline Thrombosis & $1(6.7)$ & $7(15.6)$ & 0.769 & ${ }^{\mathrm{FE}} P=0.666$ \\
\hline Hepatosplenomegaly & $2(13.3)$ & $4(8.9)$ & 0.247 & ${ }^{\mathrm{FE}} P=0.634$ \\
\hline Lymphadenopathy & $2(13.3)$ & $7(15.6)$ & 0.044 & ${ }^{\mathrm{FE}} P=1.000$ \\
\hline Osteoporosis & $1(6.7)$ & $3(6.7)$ & 0.000 & ${ }^{\mathrm{FE}} P=1.000$ \\
\hline Avascular necrosis & $1(6.7)$ & 0 & 3.051 & ${ }^{\mathrm{FE}} P=0.250$ \\
\hline
\end{tabular}

CNS, central nervous system; FE, Fisher exact; $P, P$ value for comparing between the two categories. ${ }^{\star} P \leq 0.05$, statistically significant.

Figure 2

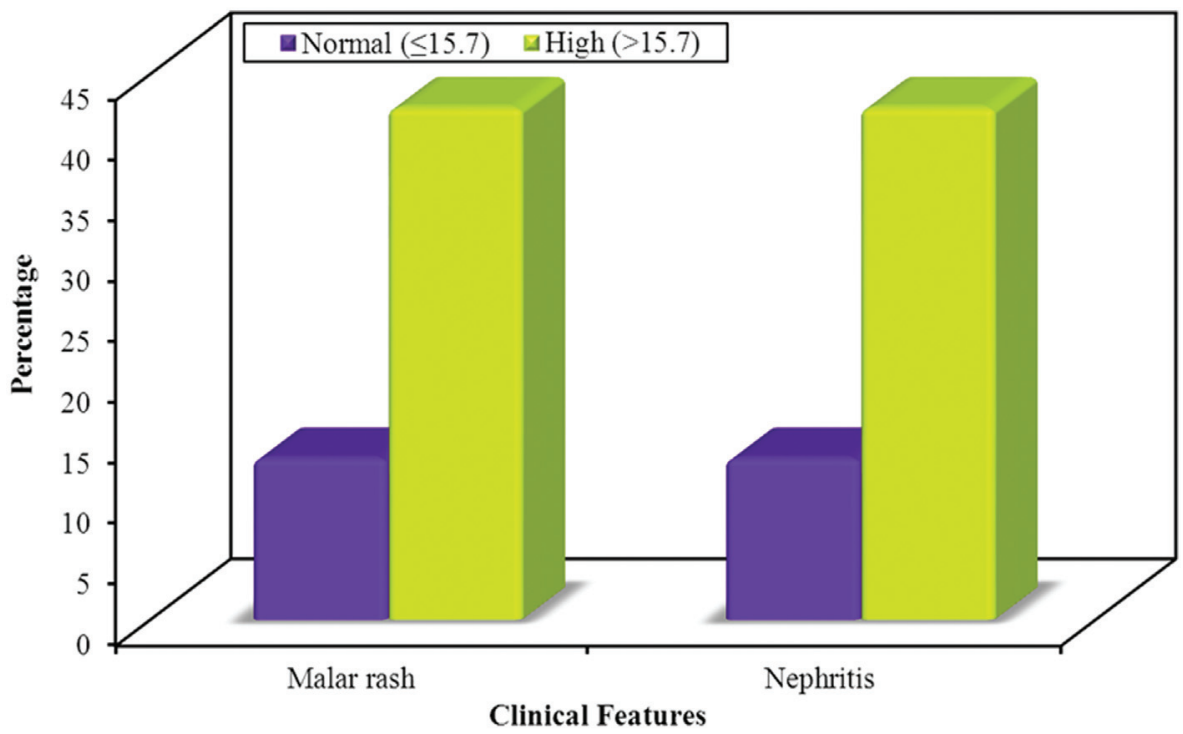

Comparison between systemic lupus erythematosus with normal serum matrix metalloproteinase-9 group and systemic lupus erythematosus with high serum matrix metalloproteinase-9 group regarding clinical features (malar rash and nephritis). 
Comparison between systemic lupus erythematosus with normal serum matrix metalloproteinase-9 group and systemic lupus erythematosus with high serum matrix metalloproteinase-9 group regarding diseases activity Systemic Lupus Activity Measure index

The diseases activity SLAM index in SLE with normal serum MMP-9 group ranged from 5 to 17 , with a mean of $10.27 \pm 3.17$. In SLE with high serum MMP-9 group, it ranged from 6 to 23, with a mean of 11.29 \pm 3.4 . There was no statistically significant difference between the two studied groups according to SLAM index (Table 4).

Comparison between systemic lupus erythematosus with normal serum matrix metalloproteinase-9 group and systemic lupus erythematosus with high serum matrix metalloproteinase-9 group regarding acutephase reactants

There was no statistically significant difference between the two studied groups according to ESR and CRP (Table 5).

Comparison between systemic lupus erythematosus with normal serum matrix metalloproteinase-9 group and systemic lupus erythematosus with high serum matrix metalloproteinase-9 group regarding immunological markers

There was no statistically significant difference between the two studied groups regarding ANA, C3, C4, and anti-dsDNA (Table 6).

Correlation between serum matrix metalloproteinase-9 level and diseases activity Systemic Lupus Activity Measure index $(n=60)$

The correlations between MMP-9 serum level and SLAM index are demonstrated in
Table 7. Significant positive correlations were detected between MMP-9 serum level and SLAM index $(r=0.270, \quad P=0.037$; Table 7 and Fig. 3).

Correlation between matrix metalloproteinase- 9 serum level and anti-double-stranded DNA $(n=60)$

The correlations between MMP-9 serum level and anti-dsDNA are demonstrated in Table 8. No significant correlations were detected between MMP-9 serum level and anti-dsDNA $(r=0.055$, $P=0.676$; Table 8 and Fig. 4).

Multiple logistic regression analysis of different immunological markers affecting peripheral neuropathy

Table 9 shows multiple logistic regression analysis of different immunological markers affecting $\mathrm{PN}$. There was a significant relation between increasing the risk of $\mathrm{PN}$ and MMP-9 serum level $(P=0.047$, odds ratio=4.031).

Receiver operating characteristic curve for optimal prediction of serum matrix metalloproteinase-9 cutoff value

ROC curve analysis showed that the optimal prediction of serum MMP-9 cutoff value was more than $15.7 \mathrm{ng} / \mathrm{ml}$, with a $71.67 \%$ sensitivity and $100.0 \%$ specificity.

Positive prediction value was 100 and negative predictive value was 54.1. The area under the curves was 0.805 , and statistically significant $P$ value was 0.001 (Table 10 and Fig. 5).

Table 4 Comparison between systemic lupus erythematosus with normal serum matrix metalloproteinase-9 group and systemic lupus erythematosus with high serum matrix metalloproteinase-9 group regarding diseases activity Systemic Lupus Activity Measure index $(n=60)$

\begin{tabular}{lccc}
\hline & \multicolumn{2}{c}{ Matrix metalloproteinase-9 } & $t$ \\
\cline { 2 - 4 } & Normal $(\leq 15.7)(n=15)$ & High $(>15.7)(n=45)$ & \\
\hline Diseases activity SLAM index & $5.0-17.0$ & $6.0-23.0$ & 1.026 \\
Range & $10.27 \pm 3.17$ & $11.29 \pm 3.40$ & \\
Mean \pm SD & & & \\
\hline
\end{tabular}

$P, P$ value for comparing between the two categories; SLAM, Systemic Lupus Activity Measure.

Table 5 Comparison between systemic lupus erythematosus with normal serum matrix metalloproteinase-9 group and systemic lupus erythematosus with high serum matrix metalloproteinase- 9 group regarding acute-phase reactants $(n=60)$

\begin{tabular}{lcc}
\hline Acute-phase reactant & \multicolumn{2}{c}{ Matrix metalloproteinase-9 } \\
\cline { 2 - 3 } & Normal $(\leq 15.7)(n=15)$ & High $(>15.7)(n=45)$ \\
\hline ESR first hour & & \\
Minimum-maximum & $21.0-135.0$ & $15.0-142.0$ \\
Median & 42.0 & 45.0 \\
CRP & $1.40-30.0$ & 0.891 \\
Minimum-maximum & 6.0 & $0.90-122.0$ \\
Median & 7.0 & 278.50 \\
\hline
\end{tabular}

CRP, C-reactive protein; ESR, erythrocytes sedimentation rate; $P, P$ value for comparing between the two categories; $U$, Mann-Whitney test. 
Table 6 Comparison between systemic lupus erythematosus with normal serum matrix metalloproteinase-9 group and systemic lupus erythematosus with high serum matrix metalloproteinase-9 group regarding immunological markers $(n=60)$

\begin{tabular}{|c|c|c|c|c|}
\hline Immunological markers & Normal $(\leq 15.7)(n=15)[n(\%)]$ & High $(>15.7)(n=45)[n(\%)]$ & Test of significance & $P$ \\
\hline \multicolumn{5}{|l|}{ ANA } \\
\hline Negative & $4(26.7)$ & $10(22.2)$ & $\chi^{2}=0.124$ & ${ }^{\mathrm{FE}} P=0.734$ \\
\hline Positive & $11(73.3)$ & $35(77.8)$ & & \\
\hline \multicolumn{5}{|l|}{ APL } \\
\hline Present & $1(6.7)$ & $7(15.6)$ & $\chi^{2}=0.769$ & ${ }^{\mathrm{FE}} P=0.666$ \\
\hline Absent & 14 (93.3) & $38(84.4)$ & & \\
\hline \multicolumn{5}{|l|}{ C3 } \\
\hline Minimum-maximum & $0.49-1.87$ & $0.13-3.71$ & $U=333.50$ & 0.946 \\
\hline Median & 1.19 & 1.25 & & \\
\hline \multicolumn{5}{|l|}{ C4 } \\
\hline Minimum-maximum & $0.07-0.47$ & $0.07-10.50$ & $U=274.50$ & 0.282 \\
\hline Median & 0.17 & 0.20 & & \\
\hline \multicolumn{5}{|l|}{ Anti-double-stranded DNA } \\
\hline Minimum-maximum & $26.0-407.0$ & $13.50-800.0$ & $U=284.0$ & 0.310 \\
\hline Median & 44.30 & 58.0 & & \\
\hline
\end{tabular}

ANA, antinuclear antibodies; APL, antiphospholipid antibodies; FE, Fisher exact; $P, P$ value for comparing between the two categories; $U$, Mann-Whitney test.

Table 7 Correlation between matrix metalloproteinase- 9 serum level and diseases activity Systemic Lupus Activity Measure index $(n=60)$

\begin{tabular}{lcr}
\hline & \multicolumn{2}{c}{ Matrix } \\
& \multicolumn{2}{c}{ metalloproteinase-9 } \\
\cline { 2 - 3 } & $r$ & $P$ \\
\hline Diseases activity SLAM index & $0.270^{*}$ & $0.037^{*}$ \\
\hline
\end{tabular}

$r$, Pearson coefficient; SLAM, Systemic Lupus Activity Measure. ${ }^{*} P \leq 0.05$, statistically significant.

\section{Discussion}

MMPs are a group of zinc-containing endoproteinases that can degrade a variety of extracellular matrix components; the up-regulation of MMP-9 within the vessel walls may be responsible for the vascular injury as a result of disturbed nerve blood-nerve barrier (BNB) permeability seen in SLE and the resulting chronic combined axonal degeneration and demyelinating type of neuropathy frequently found in SLE [13]. Thus, further studies are needed to evaluate and test the hypothesis that serum MMP-9 levels are associated with PN in patients with SLE.

The results of this study are in agreement with those conducted by Abd El-Aziz et al. [18], Ainiala et al. [11], Gurer et al. [14], Mawrin et al. [13], and FaberElmann et al. [10] which found significantly high levels of MMP-9 in patients with SLE and its relation to neuropsychiatric manifestations either centrally or peripherally, as well as its relation to lupus activity and clinical manifestations.

Abd El-Aziz et al. [18] reported that serum MMP-9 levels were found to be significantly higher $(P<0.01)$ in patients with SLE compared with the control group.
Serum MMP-9 shows statistically significant correlation with lupus nephritis, malar rash, and photosensitivity, and it did not show any statistically significant correlation with ANA, alopecia, and mucosal ulcers. In contradictory to this study, serum MMP-9 showed a statistically significant correlation with anti-dsDNA, Raynaud's phenomenon, and photosensitivity. The results proposed that MMP-9 could be involved in the pathogenesis of SLE, and serum MMP-9 can be used as a marker to monitor disease activity, renal damage, disease progression, and amelioration in SLE. However, Makowski and Ramsby [9] compared circulating MMP-9 concentrations with antibodies to ssDNA and dsDNA to determine their relation in inflammatory arthritic diseases, and patients having high antissDNA and anti-dsDNA antibodies harbored significantly lower amounts of MMP-9 when compared with patients who had low levels of both anti-ssDNA and anti-dsDNA antibodies. Hence, neutrophil MMP-9, an inflammatory marker, inversely correlates with anti-dsDNA antibodies. The presence of antibodies in patients with SLE may be a direct cause for inhibition of MMP-9 synthesis or its activity. Another conceivable clarification is that the calcium ions are hindered or destroyed, and thus activated MMP-9 is being degraded.

Gurer et al. [14] researched the expression pattern and cellular source of MMP in vasculitic neuropathy. Nerve biopsy sections of eight patients with nonsystemic vasculitic neuropathy, four with systemic vasculitic neuropathy, and eight patients with noninflammatory neuropathy were examined 


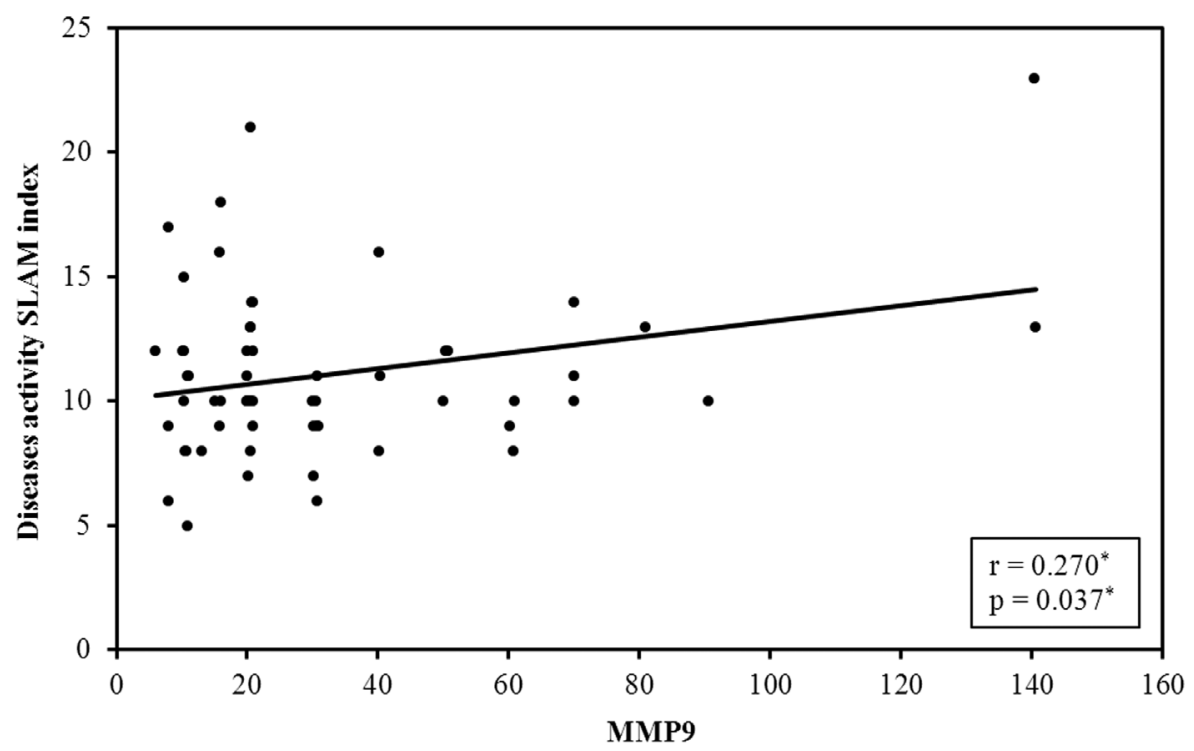

Correlation between matrix metalloproteinase-9 serum level and diseases activity Systemic Lupus Activity Measure index ( $n=60)$.

Table 8 Correlation between matrix metalloproteinase-9 serum level and anti-double-stranded DNA $(n=60)$

\begin{tabular}{lcc}
\hline & \multicolumn{2}{c}{ Matrix metalloproteinase- } \\
\cline { 2 - 3 } & $r$ & $P$ \\
\hline Anti-double-stranded DNA & 0.055 & 0.676 \\
\hline
\end{tabular}

$r$, Pearson coefficient. $P \leq 0.5$, statistically significant.

for the presence of CD4+, CD8+, and CD68+ cells and immunohistochemical analysis for MMP-2 and MMP-9 expression. No difference was recognized between immunostaining types of nonsystemic and systemic vasculitic neuropathies with the antibodies utilized, except in MMP-9 immunostaining, which was observed to be enhanced in nonsystemic vasculitic neuropathy group. These outcomes proposed a pathogenic effect of MMP-9 secreted from CD8+ cells in vasculitic neuropathy. However, Gurer et al. [14] investigated the inflammatory role of MMP-9 using nerve biopsy sample rather than the serum sample as used in this study; both agreed the pathological role of MMP-9 in vasculitic neuropathy.

Mawrin et al. [13] studied distribution pattern of MMP in PN in patients with SLE. The study investigated the expression of MMP-1, MMP-2, MMP-3, MMP-9, MMP-10, and MMP-13, and their tissue inhibitors (TIMP-1 and TIMP-2) in sural nerves from 12 patients with SLE in comparison with normal controls. It was proposed that the up-regulation of MMP-3 and MMP-9 might be a direct cause for the vascular disruption in
SLE, resulting in chronic axonal and demyelinating type of neuropathy commonly presented in SLE.

Faber-Elmann et al. [10] noticed that serum MMP-9 activity is significantly higher in patients with SLE compared with healthy controls. High serum MMP-9 activity was identified in $68 \%$ of the patients with SLE. Elevated serum MMP-9 was significantly correlated with the lupus clinical features regarding discoid rash, Raynaud's phenomenon, pneumonia, oral ulcers, and anti-phospholipid antibodies. High levels of serum MMP-9 did not show correlation with disease activity index (SLEDAI, BILAG) in female patients; however, it seems to be correlated with SLE activity in the group of male patients. The results of this work proposed that elevated serum MMP-9 level plays a significant pathogenic role in SLE.

Ainiala et al. [11] studied the association between serum (MMP-9) level and neuropsychiatric manifestations including polyneuropathy. No significant difference was detected in serum MMP-9 levels between patients with SLE and controls. On the contrary, patients with SLE who had at least one neuropsychiatric manifestation (patients with neuropsychiatric SLE) showed significantly higher serum MMP-9 level than patients with SLE without neuropsychiatric events $(P=0.009)$. Serum MMP-9 concentration did not show significant correlation with the ECLAM index as an activity monitoring; however, it was correlated significantly with the SLICC damage index. 


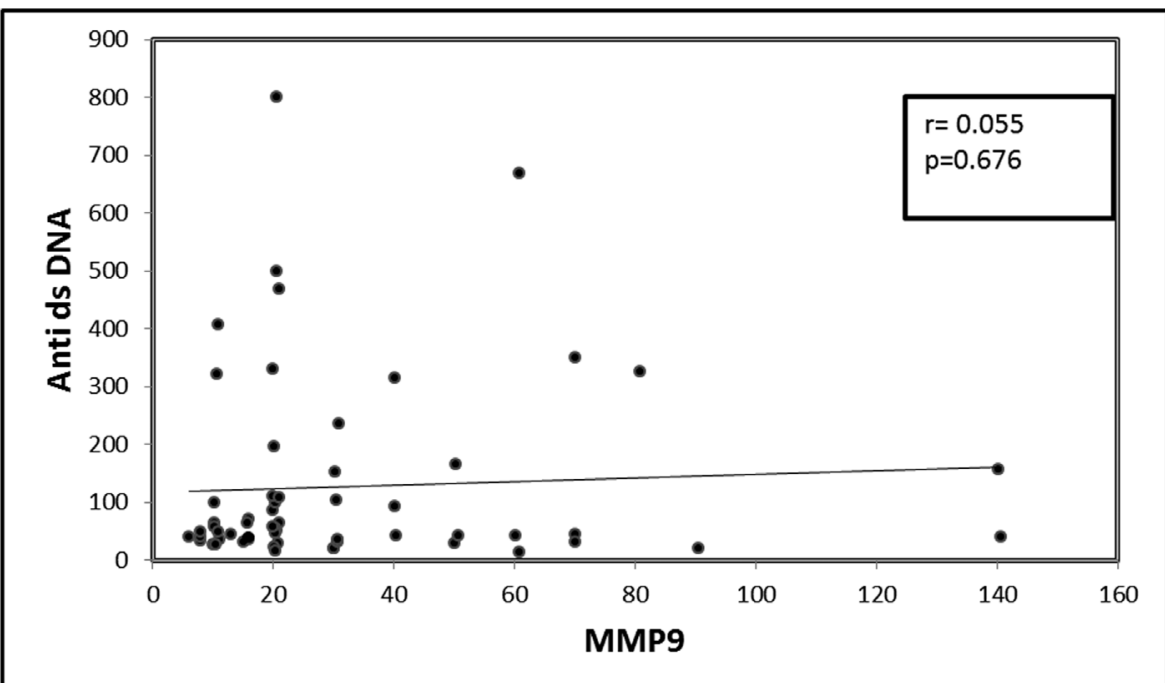

Correlation between matrix metalloproteinase-9 serum level and anti-double-stranded DNA ( $n=60)$.

Table 9 Multivariate analysis for the immunological markers affecting peripheral neuropathy

\begin{tabular}{lcccc}
\hline Immunological markers & $P$ & OR & \multicolumn{2}{c}{$95 \% \mathrm{Cl}$} \\
\cline { 3 - 5 } & & & LL & UL \\
\hline MMP-9 & $0.047^{*}$ & 4.031 & 1.297 & 16.307 \\
C3 & 0.790 & 1.165 & 0.378 & 3.587 \\
C4 & 0.601 & 1.574 & 0.288 & 8.603 \\
Anti-double-stranded DNA & 0.250 & 0.998 & 0.994 & 1.002 \\
APL & 0.790 & 0.795 & 0.146 & 4.312 \\
ANA & 0.061 & 1.007 & 1.000 & 1.015 \\
\hline
\end{tabular}

ANA, antinuclear antibodies; APL, antiphospholipid antibodies; $\mathrm{Cl}$, confidence interval; LL, lower limit; MMP, matrix metalloproteinase; OR, odds ratio; UL, upper limit. * $P \leq 0.05$, statistically significant.

Table 10 Agreement (sensitivity and specificity) for serum matrix metalloproteinase-9

\begin{tabular}{cccccc}
\hline & Cutoff & Sensitivity & Specificity & Positive prediction value & Negative predictive \\
\hline Matrix metalloproteinase-9 & $>15.7$ & 71.67 & 100.0 & 100.0 & 54.1 \\
\hline
\end{tabular}

Ertugrul et al. [8] elucidated the significance of MMP2, MMP-9, and TIMP-1 in cutaneous lupus erythematous pathogenesis; the study suggested that increased MMP-2 and MMP-9 activities may contribute to the pathogenesis of cutaneous lupus erythematous and cutaneous disease severity. In agreement with this study, malar rash as one of cutaneous lupus manifestations showed significant elevation in SLE with high MMP-9 level group.In contradictory to the results of this study, Mao et al. [19] performed a meta-analysis to estimate circulating MMP-9 levels in patients with SLE more accurately and explored its related influencing factors. The study proposed that circulating MMP-9 levels do not show significant difference between patients with SLE and healthy controls; however, the results may be affected by age and sample size. Mao et al. [19] recommended further studies to clarify the relationship between SLE and circulating MMP-9 levels. This result may be explained by younger age sample may influence the result of MMP-9 level.

In contrast to our results, Liu et al. [7] reported that decreased serum levels of MDC and MMP-9 were found in patients with renal damage than those without the damage $(P<0.001$ and $<0.05)$. These data suggested that MDC and MMP-9 might be enrolled in lupus pathogenesis and serum levels of MDC and MMP-9 could be markers of monitoring disease activity, renal involvement, disease deterioration, and improvement in SLE.

The explanation of lower serum level of MMP-9 in SLE activity and organ damage suggests its role in 


\section{Figure 5}

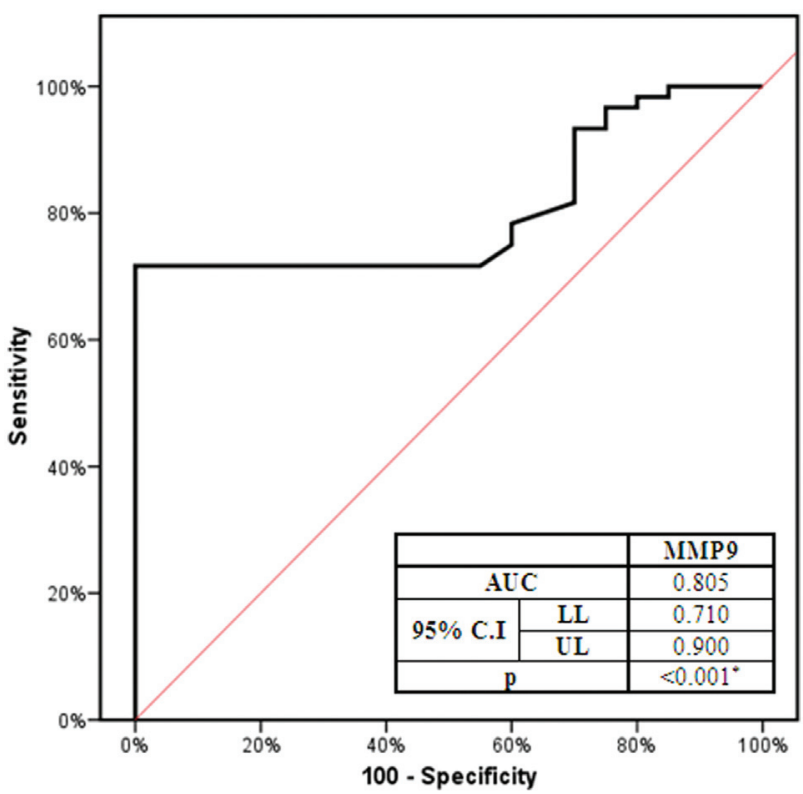

Receiver operating characteristic curve for serum matrix metalloproteinase-9.

immune complex clearance. Berzal et al. [20] proposed that MMP-9 degrades auto-antigens, coupled to immunoglobulins in IC. Further studies about the role of MMP-9 in SLE pathogenesis will suggest new and interesting information about the pathophysiology and disease progression; moreover, it will allow the possible achievement of new effective treatment options.

\section{Conclusion}

Significant elevation of serum MMP-9 may increase the risk of PN in patients with SLE. High serum MMP-9 level correlates with lupus disease activity and significantly relates to lupus nephritis and skin involvement.

\section{Financial support and sponsorship}

Nil.

\section{Conflicts of interest}

There are no conflicts of interest.

\section{References}

1 Lijnen HR. Plasmin and matrix metalloproteinases in vascular remodeling. Thromb Haemost 2001; 86:324-333.
2 Kalela A. Factors affecting serum matrix metalloproteinase 9 with special reference to atherosclerosis. Acta Univ Tamper 2002; 886:23-30.

3 Girolamo N, Indoh I, Jackson N, Wakefield D, McNeil P, Yan W, et al. Human mast cell-derived gelatinase B (matrix metalloproteinase-9) is regulated by inflammatory cytokines: role in cell migration. $\mathrm{J}$ Immunol 2006; 177:2638-2650.

4 Sharshar T, Durand MC, Lefaucheur JP, Lofaso F, Raphaël JC, Gherardi $\mathrm{RK}$, et al. MMP-9 correlates with electrophysiologic abnormalities in Guillain-Barré syndrome. Neurology 2002; 59:1649-1651.

5 Itoh T, Matsuda H, Tanioka M, Kuwabara K, Itohara S, Suzuki R. The role of matrix metalloproteinase- 2 and matrix metalloproteinase- 9 in antibodyinduced arthritis. J Immunol 2002; 169:2643-2647.

6 Fawzy RM, Abdel Hamid YA, Albehesy MM, Aboelmaged RF. Serum and synovial matrix metalloproteinase-3 as markers of disease activity in early rheumatoid arthritis. Egypt Rheumatol Rehabil 2016; 43:178-183.

7 Liu Y, Tie N, Bai L. Serum levels of MDC and MMP-9 and the relationship between serum levels and disease activity in the patients with systemic lupus erythematosus. Pak J Med Sci 2015; 31:803-806.

8 Ertugrul G, Keles D, Oktay G, Aktan S. Matrix metalloproteinase-2 and -9 activity levels increase in cutaneous lupus erythematosus lesions and correlate with disease severity. Arch Dermatol Res 2018; 310:173-179.

9 Makowski S, Ramsby L. Concentrations of circulating matrix metalloproteinase 9 inversely correlate with autoimmune antibodies to double stranded DNA: implications for monitoring disease activity in systemic lupus erythematosus. Mol Pathol 2003; 56:244-247.

10 Faber-Elmann A, Sthoeger Z, Tcherniack A, Dayan M, Mozes E. Activity of matrix metalloproteinase- 9 is elevated in sera of patients with systemic lupus erythematosus. Clin Exp Immunol 2002; 127:393-398.

11 Ainiala H, Hietaharju A, Dastidar P, Loukkola J, Lehtimäki T, Peltola J, et al. Increased serum matrix metalloproteinase 9 levels in systemic lupus erythematosus patients with neuropsychiatric manifestations and brain magnetic resonance imaging abnormalities. Arthritis Rheum 2004; 50:858-865.

12 Phillips M, Fadia M, Lea-Henry N, Smiles J, Walters D, Jiang H. MMP2 and MMP-9 associate with crescentic glomerulonephritis. Clin Kidney J 2017; 10:215-220.

13 Mawrin C, Brunn A, Röcken C, Schröder M. Peripheral neuropathy in systemic lupus erythematosus: pathomorphological features and distribution pattern of matrix metalloproteinases. Acta Neuropathol 2003; 105:365-372.

14 Gurer G, Erdem S, Kocaefe C, Ozgüç M, Tan E. Expression of matrix metalloproteinases in vasculitic neuropathy. Rheumatol Int 2004; 24:255-259.

15 Petri M, Orbai AM, Alarcon GS, Gordon C, Merrill JT, Fortin PR, et al. Derivation and validation of the Systemic Lupus International Collaborating Clinics classification criteria for systemic lupus erythematosus. Arthritis Rheum 2012; 64:2677-2686.

16 [No authors listed]. The American College of Rheumatology nomenclature and case definitions for neuropsychiatric lupus syndromes. Arthritis Rheum 1999; 42:599-608.

17 Mikdashi J, Nived O. Measuring disease activity in adults with systemic lupus erythematosus: the challenges of administrative burden and responsiveness to patient concerns in clinical research. Arthritis Res Ther 2015; 17:183.

18 Abd El-Aziz GA, Darwish HM, Morsy Amal AA, Mousa SG. Serum level of matrix metalloproteinase-9 in patients with systemic lupus erythematosus. Egypt J Hosp Med 2005; 20:1-7.

19 Mao YM, Wang S, Zhao CN, Wu Q, Dan YL, Guan SY, et al. Circulating matrix metalloproteinase- 9 levels in patients with systemic lupus erythematosus: a meta-analysis. Curr Pharm Des 2018; 24:1780-1787.

20 Berzal E, Boon L, Martens E, Vandooren J, Benedicte C, Opdenakker G. Matrix metalloproteinase-9/MMP-9/gelatinase $b$ in systemic lupus erythematosus (SLE). Lupus Sci Med 2017; 4:A54. 